\title{
Green Stimulated Emission Boosted by Nonradiative Resonant Energy Transfer from Blue Quantum Dots
}

\author{
Yuan Gao, ${ }^{\dagger, \ddagger}$ Guannan Yu, ${ }^{\dagger}$ Yue Wang, ${ }^{\dagger}$ Cuong Dang, ${ }^{\ddagger}$ Tze Chien Sum, ${ }^{\dagger}$ Handong Sun, $*^{\dagger},{ }^{\dagger}$ \\ and Hilmi Volkan Demir*, ${ }^{*},+, \S$
}

\begin{abstract}
${ }^{\dagger}$ Division of Physics and Applied Physics, School of Physical and Mathematical Sciences, Nanyang Technological University, 21 Nanyang Link, 637371, Singapore

${ }^{*}$ LUMINOUS! Center of Excellence for Semiconductor Lighting and Displays, School of Electrical and Electronic Engineering and The Photonics Institute, Nanyang Technological University, 50 Nanyang Avenue, 639798, Singapore

${ }^{\S}$ Department of Electrical and Electronics Engineering and Department of Physics, UNAM - Institute of Materials Science and Nanotechnology, Bilkent University, 06800 Bilkent, Ankara, Turkey
\end{abstract}

ABSTRACT: Thanks to their tunability and versatility, the colloidal quantum dots (CQDs) made of II-VI semiconductor compound offer the potential to bridge the "green gap" in conventional semiconductors. However, when the CQDs are pumped to much higher initial excitonic states compared to their bandgap, multiexciton interaction is enhanced, leading to a much higher stimulated emission threshold. Here, to circumvent this drawback, for the first time, we show a fully colloidal gain in green enabled by a partially indirect pumping approach assisted by Förster resonance energy transfer process. By introducing the blue CQDs as exciton donors, the lasing threshold of the green CQDs, is reduced dramatically. The blue CQDs thus serve as an energy-transferring buffer medium to reduce excitation energy from pumping photons in a controlled way by injecting photoinduced excitons into green CQDs. Our newly developed colloidal pumping scheme could enable efficient CQD lasers of full visible colors by a single pump source and cascaded exciton transfer. This would potentially pave the way for an efficient multicolor laser for lighting and display applications.

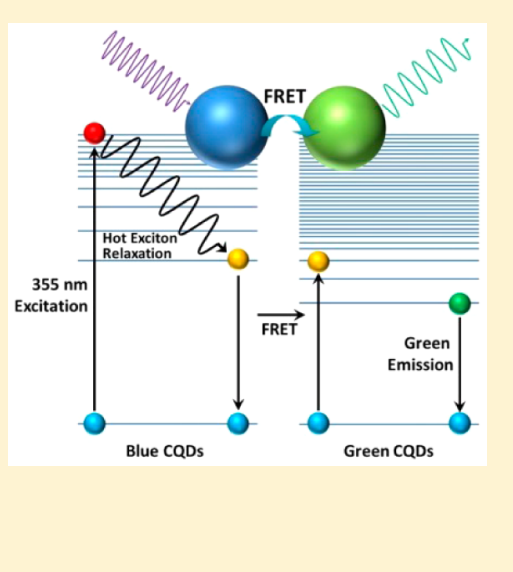

$\mathrm{T}$ raditional bulk semiconductor laser materials are unable to cover the full visible spectral range with a decent energy efficiency. The emission from III-V nitride semiconductors in green regime suffers the so-called "green gap" because of the energy inefficiency in the green regime. ${ }^{1}$ On the other hand, in light of the emission color tunability of colloidal quantum dots (CQDs) by adjusting their size, structure, and composition, CQDs can be a fabulous material system that is able to provide optical gain at virtually any visible and near-infrared wavelengths if they are carefully designed. ${ }^{2,3}$ Meanwhile, the atomiclike energy level of CQDs guarantees their laser performance stable and temperature insensitive. ${ }^{4,5}$ Stimulated emission from CQDs was first observed in 1991 from CdSe nanocrystals that were doped in glass and optically pumped at liquid nitrogen temperature. ${ }^{6}$ Since Klimov et al. demonstrated optical gain from CdSe CQDs at room temperature, ${ }^{4,7}$ the studies of lasing from CQDs have been on a rapid rise. Researchers have shown optical gain from blue to red emitting CQDs, which covers the full visible spectrum. ${ }^{8,9}$ Therefore, CQDs, with the properties of tunable wavelength and high fluorescence quantum yield, make them promising to address the "green gap" problem.

Given the current optical pump mechanism, a useful CQD laser device requires a practical pumping source rather than ultrashort pulsed Ti-Saphire lasers. The standard and commonly adopted pump wavelengths are 532 and 355 $\mathrm{nm},{ }^{10}$ which are easy to access as the second and third

harmonic wavelength of Nd:YAG laser (fundamental wavelength of $1064 \mathrm{~nm}$ ). Laser of $532 \mathrm{~nm}$ is only capable of pumping CQDs of long wavelength emission. Thus, the standard wavelength of $355 \mathrm{~nm}$ should be used for optical pumping CQDs to cover the full visible spectrum. However, the development of optical gain in CQDs is very sensitive to the initial excitonic states that are populated. ${ }^{11,12}$ Therefore, the lasing threshold actually depends on the wavelengths of CQD emission and the pumping source. Consequently, CQDs that lase at different wavelengths should have different optimum pump wavelengths, and the standard wavelength $355 \mathrm{~nm}$ (albeit commonly adopted) cannot be the universal best pumping source for CQDs.

To overcome the drawback outlined above, we developed, for the first time, a partially indirect pumping approach assisted by Förster resonance energy transfer (FRET) to excite CQDs optically. Although the quality of the green CQD films was excellent, the optical gain was not possible to observe by direct pumping with our existing laser of $355 \mathrm{~nm}$. However, in the sample with the mixed solution of blue and green CQDs, we achieved green CQD lasing, optically pumped by the standard wavelength of $355 \mathrm{~nm}$ under the same laser configuration. The

Received: May 23, 2016

Accepted: July 7, 2016

Published: July 7, 2016 
time-resolved photoluminescence of green and blue CQDs were measured, respectively, in the presence or absence of the other. The decay dynamics of blue CQDs, acting as donors, and green CQDs, serving as acceptors, confirm FRET process takes place between these two types of CQDs. Therefore, the critical process is exciton relaxation in the blue CQDs, and then the energy was resonantly transferred to the green CQDs. This avoids populating the green CQDs with all very high energy excitons corresponding to the $355 \mathrm{~nm}$ pump laser. We note that the green CQDs were still partially excited by direct laser absorption. Our partially indirect pump scheme assisted by FRET to enable green CQD lasing can be used for other CQDs emitting at different visible wavelengths by a universal single pumping laser wavelength.

The CdZnSe/ZnS blue and $\mathrm{CdSe} / \mathrm{ZnS}$ green CQDs were synthesized using one pot method according to published literature. $^{13-15}$ The as-synthesized blue and green emitting CQDs show a very narrow PL spectrum with full-width-at-halfmaximum (fwhm) of 22 and $18 \mathrm{~nm}$, as presented in Figure 1a.
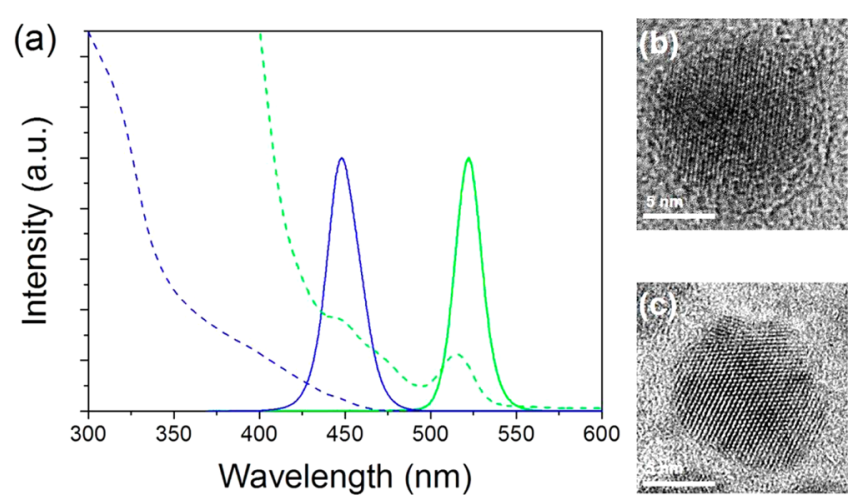

Figure 1. (a) Normalized absorption (dash line) and PL (solid line) spectra of diluted green (green curves) and blue (blue curves) emitting CQD solutions. $(b, c)$ HRTEM images of a single green and blue emitting CQD, respectively. The scale bar of both $b$ and $c$ is $5 \mathrm{~nm}$.

The fluorescence quantum yield of the blue and green CQDs in toluene solution is 0.550 and 0.845 , which was determined by using an integrating sphere at the excitation wavelength of 405 $\mathrm{nm}$. The narrow emission and high fluorescence quantum yield of the green CQDs imply their narrow size dispersity and high crystal quality. The transmission electron microscopy (TEM) images of spherical green and blue CQDs are shown in Figure $1 b, c$, and the thick shell guarantees a good passivation of the core. The emission spectrum of the blue CQDs overlaps with the absorption bands of the green CQDs to allow nonradiative energy transfer from blue to green CQDs. ${ }^{16}$

The solution with a high concentration of blue or green CQDs $(100 \mathrm{mg} / \mathrm{mL})$ was loaded into a capillary tube with the diameter of $300 \mu \mathrm{m}$, and the CQD solution was dried under reduced pressure to form a densely packed CQD superlattice. The capillary tube with a diameter of hundreds micrometer not only can serve as a container of the gain medium, but also can act as a cylindrical optical microcavity, which can support multi modes of lasing that cover the full range of the gain profile of CQDs. ${ }^{17-19}$ In addition, only a tiny amount of gain medium solution, less than $10 \mu \mathrm{L}$, is needed to be loaded in a capillary tube in contrast to the quantity that is required to make a thick and uniform spin-casted film on a glass slide for stripe excitation measurement. Therefore, gain medium in such a capillary tube is an excellent laser configuration for CQD gain medium characterization.

The samples were optically pumped with a pulsed UV laser at $355 \mathrm{~nm}$ wavelength (the third harmonic of a Nd: YAG laser) with a repetition rate of $60 \mathrm{~Hz}$ and pulse width of 520 ps. Compared to a pulse laser with a pulse duration in the order of femtoseconds, a pulsed Nd:YAG laser with nanosecond pulse width is more commonly used with cost advantages as an optical pumping source. In this work, because the pulse duration of the optical pumping source is in subnanosecond range, which is much larger than the typical time constant of Auger recombination of multiexcitons (sub 100 ps for small cores), the CQDs were pumped in a quasi-continuous wave regime. ${ }^{8,20-22}$

When the neat blue CQDs in the capillary tube were optically pumped, a sharp peak emerged at the low energy side of the spontaneous emission, as shown in Figure 2a. The whispering gallery modes (WGMs) cannot be resolved here because the resolution of our monochromator is around 0.6 $\mathrm{nm}$, but the free spectral range of the WGM lasing in this laser configuration is expected to be about $0.2 \mathrm{~nm}$ (calculated from the diameter of the capillary tube and the refractive index of the CQD film). ${ }^{18}$ A clear lasing threshold is exhibited in the laser transfer function that is plotted in Figure $2 \mathrm{~b}$. However, if we replaced the blue CQDs by the green CQDs under the exactly same pumping conditions, the green CQDs only showed spontaneous emission, Figure 2c. As can be seen in Figure 2d, the emission intensity of the green CQDs built up quite linearly with the increase of pumping fluence during the initial stage of optical pumping, but the rate of the emission intensity increment reduced under high excitation fluence. The slope of the green CQD emission intensity as the function of pump energy decreased by $43.4 \%$ when they were pumped by pulses stronger than $25 \mu \mathrm{J}$ compared to that of being pumped with low energy pulses. Thus, the PL quantum yield dropped from 0.845 under weak excitation to 0.478 under strong optical pumping. When the green CQDs are strongly pumped, multiexcitons will be generated in the CQDs, and, as a consequence, Auger recombination will take place. ${ }^{21-23}$ This nonradiative multiexciton recombination process with very short lifetime $(\sim 100 \mathrm{ps})^{8}$ outrates the radiative spontaneous emission (lifetime of $7.66 \mathrm{~ns}$ ). Therefore, the PL quantum yield of the green CQDs decreased with increasing the pulse energy of the pumping laser. On the other hand, since the photon energy of the excitation source is $3.49 \mathrm{eV}$ (corresponding to $355 \mathrm{~nm}$ ), the resulting excitons have much higher energy compared to the bandgap of the green CQDs, which is $2.34 \mathrm{eV}$ (corresponding to $529 \mathrm{~nm}$ ). Therefore, the Auger process that assists hot electrons' relaxation to the band edge excites the holes into very high energy levels, which results in a high probability of the hole trapping at the surface, interface, or high energy defects of the green CQDs. ${ }^{24-28}$ Therefore, the population inversion at the band gap can be impeded, and the laser threshold can be extremely high for the green CQDs. More importantly, the higher initial excitonic levels are subjected to higher order state degeneracy that enables multiexciton interaction, leading to progressive higher multiexciton binding energy. Figure 3 shows the transient absorption (TA) spectra of diluted green CQD solution. $\Delta \alpha(t, \lambda)=\alpha(t, \lambda)$ $-\alpha 0(\lambda)$, where $\alpha(t, \lambda)$ is absorption spectrum at the time $t$ after ultrashort excitation, and $\alpha 0(\lambda)$ is the absorption spectrum in the absence of the pump excitation. ${ }^{29}$ The excitonic peak at around $515 \mathrm{~nm}$ manifests as a photobleaching or negative $\Delta \alpha$ 

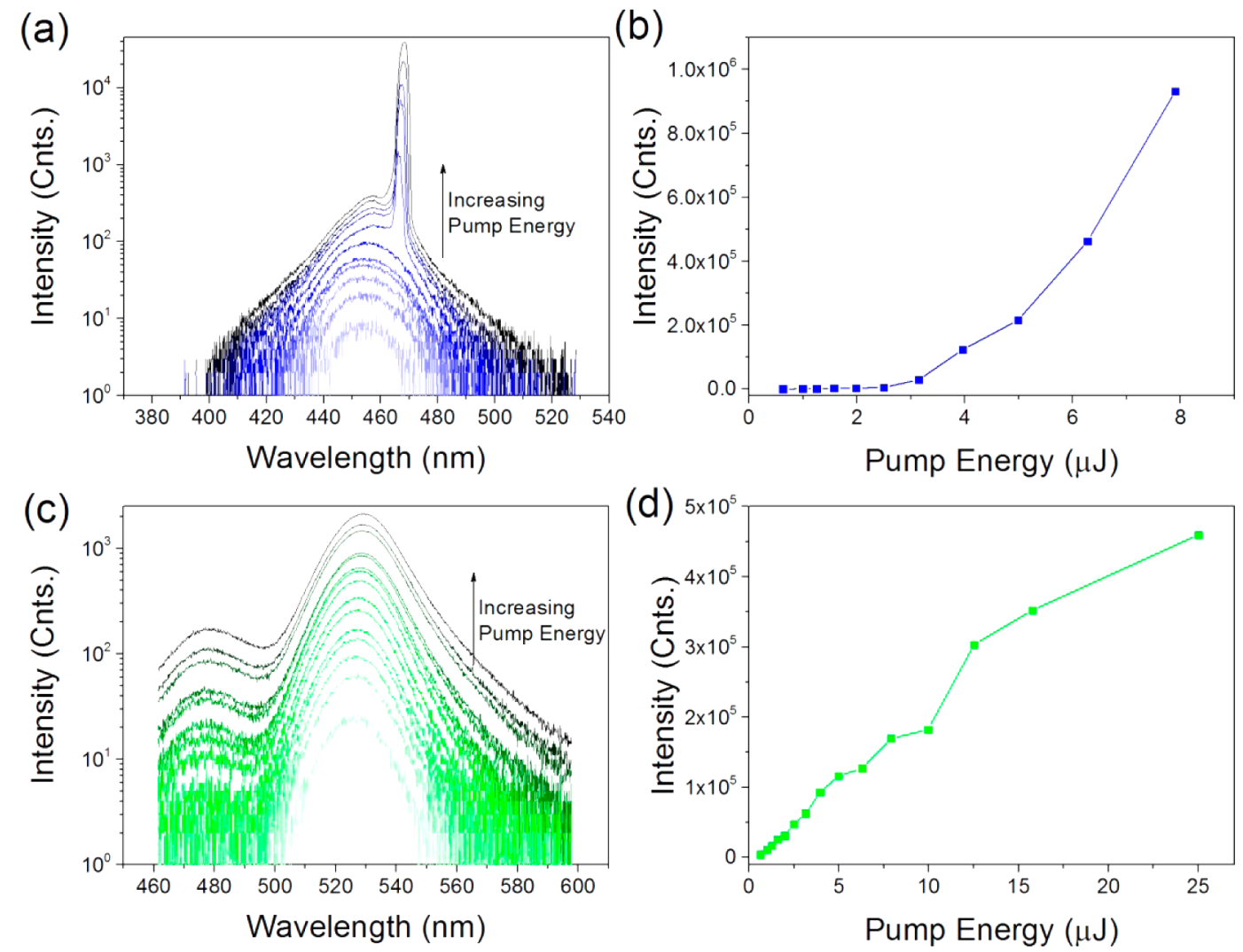

Figure 2. Evolution of spectra for blue (a) and green (c) emitting CQD layer in the capillary tubes with increasing pump power. The integrated intensity of blue (b) and green (d) CQD emission as a function of pumping energy.

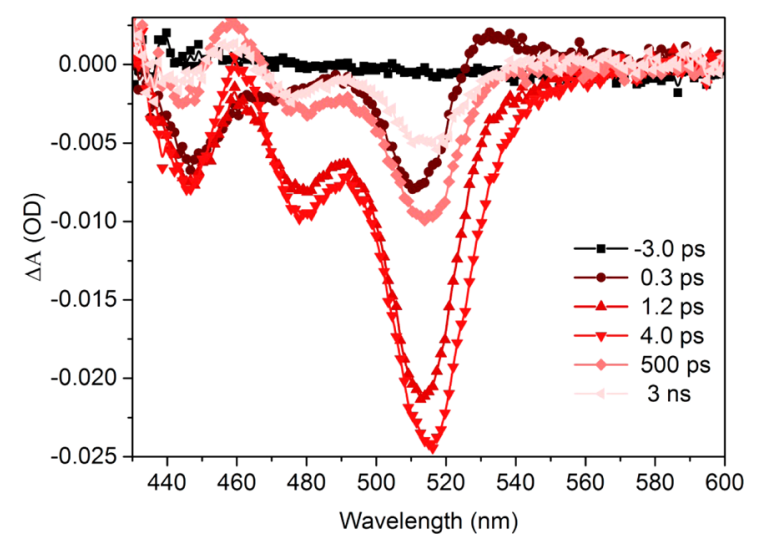

Figure 3. Transient absorption spectra of green CQD solution that was pumped at $355 \mathrm{~nm}$ (pump fluence $\sim 10 \mu \mathrm{J} / \mathrm{cm}^{2}$ ). The data was collected using a Helios system driven by a femtosecond Ti-sapphire laser.

peak in the TA spectra. This photobleaching peak rises within the first few picoseconds and then falls as the excitons undergoes recombination. This excitonic feature exhibiting a typical red shift with time is consistent with multiexciton behavior (i.e., increased multiexciton binding energy) in a typeI quantum well. ${ }^{26}$ Such higher multiexciton binding energy ushers in a broadened gain width, reduced stimulated emission cross-section and enhanced photoinduced absorption at the band edge. ${ }^{11,12,24}$ As a result, the threshold of optical gain for such high energy excited CQDs could be increased dramatically, and usually the optical gain is blocked in this case. ${ }^{11}$ In contrast, when the same sample of green CQDs was optically pumped by another pulsed laser at a wavelength of $460 \mathrm{~nm}$ and with a pulse width of $5 \mathrm{~ns}$, the sample starts to lase under a low pump energy (threshold of $2 \mu \mathrm{J}$ ), as shown in Figure 4. It is worth to note that the pulse duration ( $5 \mathrm{~ns})$ is much longer than the laser pulse that was used for previous pumping experiments with the wavelength of $355 \mathrm{~nm}$ and pulse width of $520 \mathrm{ps}$. When the photon energy of the pumping source decreases from $3.49 \mathrm{eV}$ (355 nm wavelength) to $2.69 \mathrm{eV}$ (460 $\mathrm{nm}$ wavelength), the amount of excess energy that needed to be released for relaxation to the band edge was $23 \%$ smaller, which made the CQD less heated and holes relax faster to the band edge without much exciting trap states due to Auger process. ${ }^{30}$ Moreover, when the sample was pumped at $460 \mathrm{~nm}$, the multiexciton binding energy was much smaller compared to that under $355 \mathrm{~nm}$ pumping, and the effect of optical gain blocking due to high initial excitonic states could be mitigated. ${ }^{11,12}$ Therefore, the optical gain could be observed from the green CQDs at the pumping wavelength of $460 \mathrm{~nm}$.

Systematic experiments were conducted with the CQD mixture of different weight ratios in amounts of blue to green CQDs in a capillary tube. The spherical green and blue CQDs that are used in our experiments exhibit a similar diameter (Figure 1b,c); closely packed CQD film of the mixture of blue and green CQDs can be formed. The capillary tubes were loaded with mixed CQDs in toluene solution with concentrations of blue/green CQDs of $83 \mathrm{mg} / 83 \mathrm{mg}$ per $\mathrm{mL}$ (1:1), 83 $\mathrm{mg} / 41.5 \mathrm{mg}$ per $\mathrm{mL}(2: 1), 83 \mathrm{mg} / 27.6 \mathrm{mg}$ per $\mathrm{mL}(3: 1)$, respectively. The mixed CQD solution was loaded in capillary tubes with a diameter of $300 \mu \mathrm{m}$ by capillary action. The CQD solution in the capillary tubes was dried under reduced 

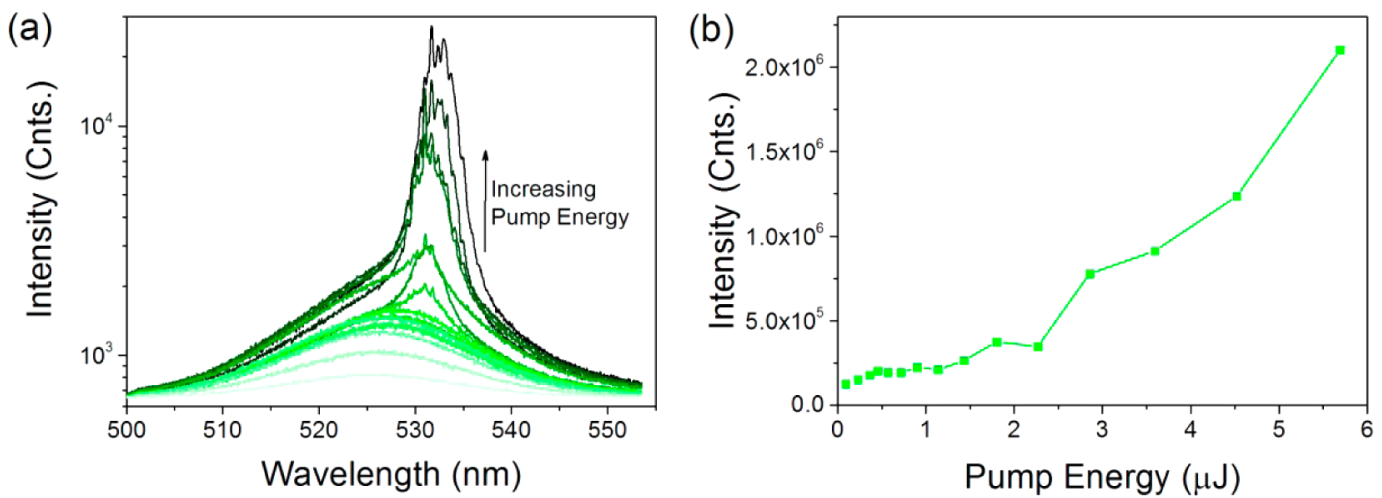

Figure 4. Green CQDs pumped by a $460 \mathrm{~nm}$ wavelength laser with a $5 \mathrm{~ns}$ pulse width: (a) spectral evolution of the green CQDs in the capillary tube as the pump energy increased; (b) laser transfer function of the green CQD layer in the capillary tube.

(a)

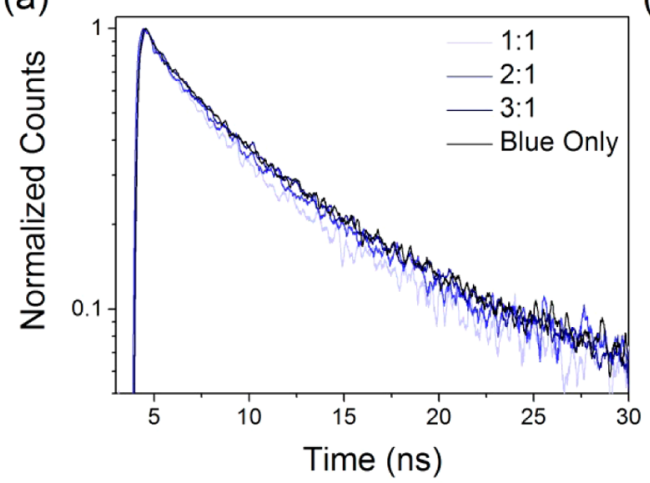

(b)

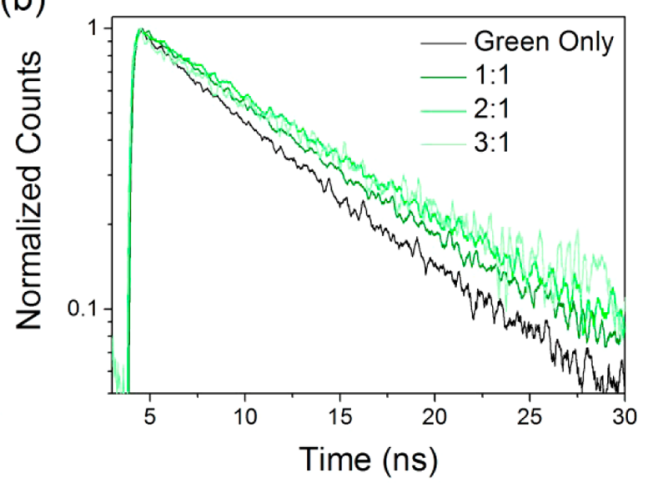

Figure 5. (a) Normalized photoluminescence decay of the blue emission from the blue emitting CQD layer (black curve), the mixture of the blueand green-emitting CQDs with mass ratios of 3:1, 2:1, and 1:1. (b) Normalized photoluminescence decay of the green emission from the greenemitting CQD layer (black curve), the mixture of the blue- and green-emitting CQDs with mass ratios of 1:1, 2:1, and 3:1.

pressure. A closely packed binary CQD layer was formed attaching to the inner wall of the capillary tubes. ${ }^{31}$

To determine the fluorescence lifetime of the blue and green emission from the CQD mixture, the samples were excited with a solid-state pulse laser diode at a wavelength of $375 \mathrm{~nm}$ and with a repetition rate of $20 \mathrm{MHz}$ under low excitation power, as shown in Figure 5. The decay curve was fitted by a single exponential function, and the lifetime constants of fluorescence from the samples with different ratios of blue and green CQDs are listed in Table 1. In light of the thick multishells of the CQDs and the long aliphatic chains of the ligands that passivate them, the charge transfers between CQDs are inhibited, ${ }^{32,33}$ while their core-to-core distance is within the range of nonradiative resonant energy transfer. The fluorescence lifetime of the donors (blue CQDs, monitored at $456 \mathrm{~nm}$ ) decreased from $7.43 \mathrm{~ns}$ for pure blue CQDs to $5.88 \mathrm{~ns}$ when the portion

Table 1. Summary of the Single Exponential Function Fitted Decay Lifetime of the Donor Blue CQDs and the Acceptor Green CQDs in the CQD Mixture with Different Blue/ Green Ratios

$\begin{array}{lcc}\text { blue donor:green acceptor } & \text { donor }(\mathrm{ns}) & \text { acceptor }(\mathrm{ns}) \\ \text { green CQDs only } & - & 7.662 \\ 1: 1 & 5.880 & 8.509 \\ 2: 1 & 6.743 & 9.702 \\ 3: 1 & 7.350 & 9.916 \\ \text { blue CQDs only } & 7.426 & -\end{array}$

of blue/green was equal (1:1). In addition, the lifetime constant of donors increased with increasing donor concentration. Meanwhile, the lifetime of the acceptor emission (green emission) changed from $7.66 \mathrm{~ns}$ in the absence of donors (blue CQDs) to $9.12 \mathrm{~ns}$ when the ratio between blue and green CQDs was 3:1. Moreover, the higher concentration of the donor is, the larger lifetime constant of the acceptor becomes. The experimental results confirmed FRET process took place from the donors (blue CQDs) to the acceptors (green CQDs). The FRET process transfers excitation energy from the blue CQDs to the green CQDs and potentially supports optical gain of the green CQDs.

As shown in Figure 6a, when the sample of the mixed blue and green CQDs with the ratio of 1:1 was optically pumped by the pulsed laser at the wavelength of $355 \mathrm{~nm}$, with increasing pumping power, lasing peaks emerged on the low energy side of the spontaneous emission spectrum of the green CQDs. By contrast, the sample with only green CQDs did not lase with exactly the same optical pumping configuration even under the highest power that the pumping laser can reach (Figure 2c). In the sample of the mixed blue and green CQDs with the ratio of $1: 1$, the blue CQDs were able to excite the green CQDs indirectly via FRET and reabsorption process at the emission wavelength of the blue CQDs that is around $456 \mathrm{~nm}$. So, this implies that a portion of excitons that contribute to the stimulated emission in the green CQDs did not release their all extra energy in the green CQDs but partially in the blue ones. Therefore, the green CQDs were less hot with the present of blue CQDs because of the indirect pumping sources and 
(a)

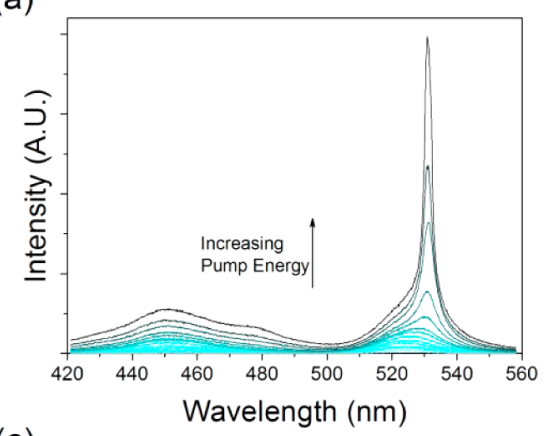

(c)

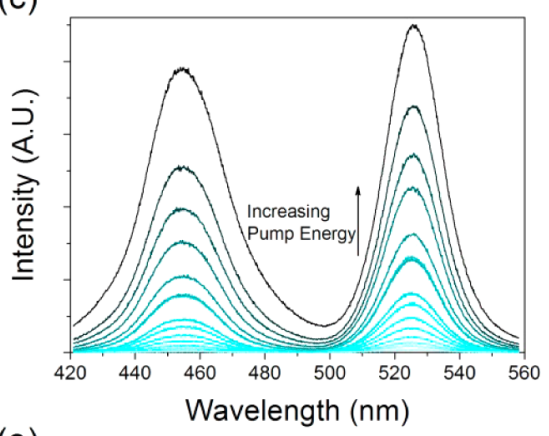

(e)

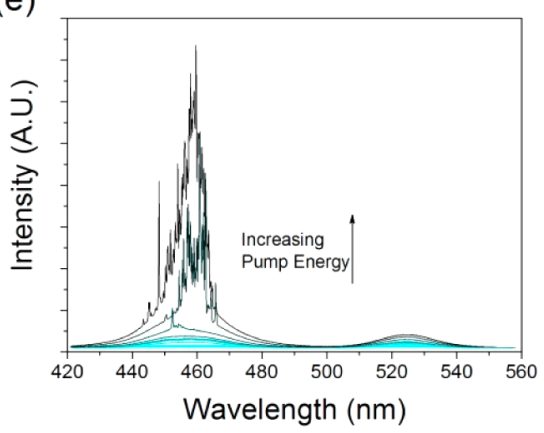

(b)

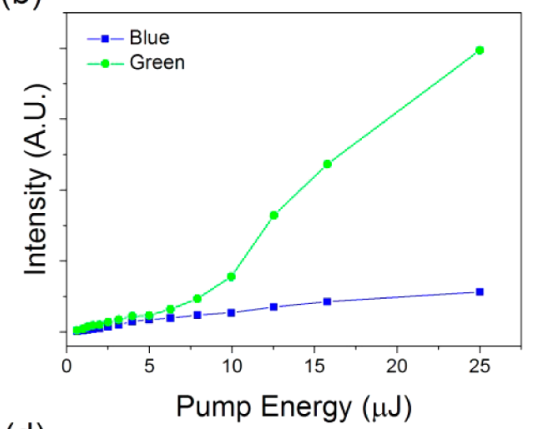

(d)

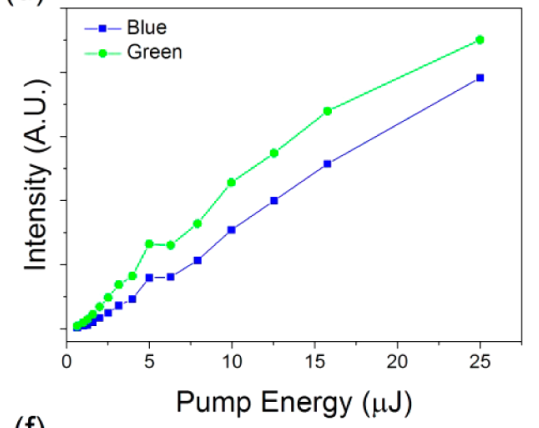

(f)

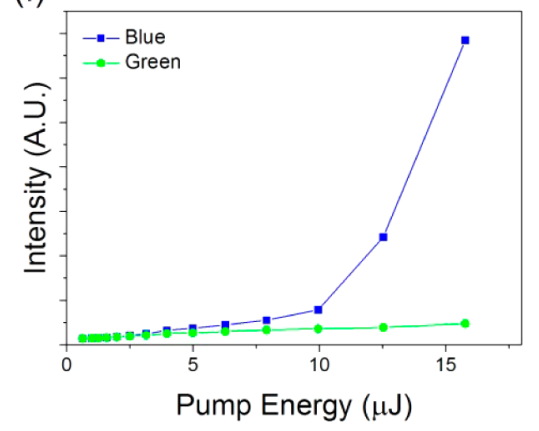

Figure 6. (a,f) Emission spectral evolution $(\mathrm{a}, \mathrm{c}, \mathrm{e})$ and the integrated intensity as a function of pumping energy (b,d,f) for mixed blue and green emitting CQDs in the capillary tube with the mass ratio of blue to green emitting CQDs of 1:1 (a,b), 2:1 (c,d), and 3:1 (e,f).

thermal buffer. On the other hand, the blue CQDs that serve as the indirect pumping source exhibited only spontaneous emission unlike their behavior in the only blue CQD sample. Because a portion of the excitons that were formed in the blue CQDs transferred to the green CQDs via nonradiative channels, the population inversion in the blue CQDs would be hindered. Moreover, the photons emitted from the blue CQDs were lost because of reabsorption by the green CQDs, which is not desirable for optical amplification process. Meanwhile, the concentration of the blue CQDs in this mixture was low so that there was not enough gain for their laser action.

When the mixture of blue and green CQDs with a portion of 2:1 in weight, as shown in Figure 6c,d, even at the highest level of our optical pumping source, both blue and green CQDs only showed spontaneous emission. The acceptors were surrounded by the donors, and more excitons were transferred to the green CQDs, which is confirmed by the prolonged fluorescence decay of the green CQD acceptor in the mixture sample with blue/ green ratio of 2:1. Moreover, more excitons could be relaxed in the blue CQDs making the green CQDs "cooler". However, the green CQDs did not lase even with the sample was pumped by the highest energy pulse available. The main reason is that the concentration of the green CQDs was not high enough to provide sufficient optical gain. In addition, due to the reabsorption of blue photons and the energy transfer from the blue CQDs to the green ones, the population inversion in the blue CQDs was impeded significantly. If the ratio of the blue to green CQDs was increased to 3:1, Figure 6e and 6f, only the blue CQDs started to lase under high optical fluence. In this case, the concentration of the blue CQDs in the mixture was large enough to support optical gain in this medium. Moreover, the spectral range of stimulated emission was broader compared to that of the pure blue CQDs that is shown in Figure 2a. Mixing green CQDs with blue ones increased the lasing threshold of blue dots dramatically. Thus, blue CQDs only lase under very high pump fluence, which generated more excitons in them. Therefore, the enhanced multiexciton interaction led to a much broadened gain profile. ${ }^{11}$

In conclusion, for the first time, we have demonstrated that using the blue CQDs as exciton donors reduce dramatically the lasing threshold of the green CQDs via FRET process under optical pumping at $355 \mathrm{~nm}$ in a quasi-continuous wave regime. If the green CQDs are initially pumped to high excitonic levels, given the stronger multiexciton interaction and high exciton energy, the gain profile of the CQDs can be broadened, the stimulated emission cross-section can be reduced and a higher probability of hole trapping can be expected. Therefore, the 
lasing threshold of the green CQDs is significantly increased when they are directly pumped by a short wavelength laser (such as at $355 \mathrm{~nm}$ ). The partially indirect optical pump approach with a practical pumping laser source circumvents the limitation of directly populating a large number of excitons at the high excitonic states of the green CQDs. The blue CQDs work as exciton providers that absorb the high pumping energy to create exciton and then transfer exciton energy to lower energy levels of the green CQDs. Our results indicate that the performance of a CQD-based laser depends heavily on their initial excitonic states, and the proposed indirect pump approach demonstrates a simple way to achieve efficiently multicolor CQD lasers with a single standard optical pumping source.

\section{AUTHOR INFORMATION}

\section{Corresponding Authors}

*E-mail: hdsun@ntu.edu.sg (H.D.S.).

*E-mail: hvdemir@ntu.edu.sg (H.V.D.).

\section{Notes}

The authors declare no competing financial interest.

\section{ACKNOWLEDGMENTS}

The authors would like to thank the financial support from Singapore National Research Foundation under NRF-CRP-62010-02 and NRF-NRFI2016-08. We would like to thank Tao Ding and Dr. Kah Ee Fong for providing CQDs that were synthesized at Luminous! Centre of Excellence for Semiconductor Lighting and Displays. The electron microscopy imaging was performed at the Facility for Analysis, Characterization, Testing and Simulation (FACTS) at Nanyang Technological University, Singapore. Financial support from Nanyang Technological University start-up grants M4080514 and M4081482, the Ministry of Education AcRF Tier 1 grants RG 9215, RG 101/15, RG 70/15 and Tier 2 grants MOE2013T2-1-081 and MOE2014-T2-1-044; the Singapore National Research Foundation (NRF) through the Singapore-Berkeley Research Initiative for Sustainable Energy (SinBeRISE) CREATE Program and the Competitive Research Program NRF-CRP14-2014-03 is also gratefully acknowledged. HVD gratefully thanks TUBA.

\section{REFERENCES}

(1) Coleman, J. J.; Bryce, A. C.; Jagadish, C. Advances in Semiconductor Lasers; Academic Press: San Diego, CA, 2012.

(2) Talapin, D. V.; Lee, J.-S.; Kovalenko, M. V.; Shevchenko, E. V. Prospects of Colloidal Nanocrystals for Electronic and Optoelectronic Applications. Chem. Rev. 2010, 110, 389-458.

(3) Gaponenko, S.; Demir, H. V.; Seassal, C.; Woggon, U. Colloidal Nanophotonics: The Emerging Technology Platform. Opt. Express 2016, 24, A430-A433.

(4) Klimov, V.; Mikhailovsky, A.; Xu, S.; Malko, A.; Hollingsworth, J.; Leatherdale, C.; Eisler, H.-J.; Bawendi, M. Optical Gain and Stimulated Emission in Nanocrystal Quantum Dots. Science 2000, 290, 314-317.

(5) Asada, M.; Miyamoto, Y.; Suematsu, Y. Gain and the Threshold of Three-Dimensional Quantum-Box Lasers. IEEE J. Quantum Electron. 1986, 22, 1915-1921.

(6) Vandyshev, Y. V.; Dneprovskii, V.; Klimov, V. Manifestation of Dimensional Quantization Levels in the Nonlinear Transmission Spectra of Semiconductor Microcrystals. JETP Lett. 1991, 53, 314318.

(7) Klimov, V. I.; Bawendi, M. G. Ultrafast Carrier Dynamics, Optical Amplification, and Lasing in Nanocrystal Quantum Dots. MRS Bull. 2001, 26, 998-1004.
(8) Dang, C.; Lee, J.; Breen, C.; Steckel, J. S.; Coe-Sullivan, S.; Nurmikko, A. Red, Green and Blue Lasing Enabled by Single-Exciton Gain in Colloidal Quantum Dot Films. Nat. Nanotechnol. 2012, 7, 335-339.

(9) Neumann, A.; Wierer, J. J.; Davis, W.; Ohno, Y.; Brueck, S. R.; Tsao, J. Y. Four-Color Laser White Illuminant Demonstrating High Color-Rendering Quality. Opt. Express 2011, 19, A982-A990.

(10) Cerdán, L.; Enciso, E.; Martín, V.; Bañuelos, J.; López-Arbeloa, I.; Costela, A.; García-Moreno, I. Fret-Assisted Laser Emission in Colloidal Suspensions of Dye-Doped Latex Nanoparticles. Nat. Photonics 2012, 6, 623-628.

(11) Cooney, R. R.; Sewall, S. L.; Sagar, D.; Kambhampati, P. Gain Control in Semiconductor Quantum Dots Via State-Resolved Optical Pumping. Phys. Rev. Lett. 2009, 102, 127404.

(12) Cooney, R. R.; Sewall, S. L.; Sagar, D.; Kambhampati, P. StateResolved Manipulations of Optical Gain in Semiconductor Quantum Dots: Size Universality, Gain Tailoring, and Surface Effects. J. Chem. Phys. 2009, 131, 164706.

(13) Lee, K.-H.; Lee, J.-H.; Song, W.-S.; Ko, H.; Lee, C.; Lee, J.-H.; Yang, H. Highly Efficient, Color-Pure, Color-Stable Blue Quantum Dot Light-Emitting Devices. ACS Nano 2013, 7, 7295-7302.

(14) Shen, H.; Bai, X.; Wang, A.; Wang, H.; Qian, L.; Yang, Y.; Titov, A.; Hyvonen, J.; Zheng, Y.; Li, L. S. High-Efficient Deep-Blue LightEmitting Diodes by Using High Quality Znxcd1-Xs/Zns Core/Shell Quantum Dots. Adv. Funct. Mater. 2014, 24, 2367-2373.

(15) Lee, K.-H.; Lee, J.-H.; Kang, H.-D.; Park, B.; Kwon, Y.; Ko, H.; Lee, C.; Lee, J.; Yang, H. Over 40 Cd/a Efficient Green Quantum Dot Electroluminescent Device Comprising Uniquely Large-Sized Quantum Dots. ACS Nano 2014, 8, 4893-4901.

(16) Lakowicz, J. R. Principles of Fluorescence Spectroscopy; Springer: New York, 2007.

(17) Wang, Y.; Leck, K. S.; Ta, V. D.; Chen, R.; Nalla, V.; Gao, Y.; He, T.; Demir, H. V.; Sun, H. Blue Liquid Lasers from Solution of Cdzns/Zns Ternary Alloy Quantum Dots with Quasi-Continuous Pumping. Adv. Mater. 2015, 27, 169-175.

(18) Gao, Y.; Zhao, X.; Wang, Y.; Chen, R.; Mutlugun, E.; Fong, K. E.; Tan, S. T.; Dang, C.; Sun, X. W.; Sun, H.; et al. Observation of Polarized Gain from Aligned Colloidal Nanorods. Nanoscale 2015, 7, 6481-6486.

(19) Kazes, M.; Lewis, D. Y.; Ebenstein, Y.; Mokari, T.; Banin, U. Lasing from Semiconductor Quantum Rods in a Cylindrical Microcavity. Adv. Mater. 2002, 14, 317-321.

(20) Klimov, V.; Mikhailovsky, A.; McBranch, D.; Leatherdale, C.; Bawendi, M. Quantization of Multiparticle Auger Rates in Semiconductor Quantum Dots. Science 2000, 287, 1011-1013.

(21) Dang, C.; Lee, J.; Roh, K.; Kim, H.; Ahn, S.; Jeon, H.; Breen, C.; Steckel, J.; Coe-Sullivan, S.; Nurmikko, A. Highly Efficient, Spatially Coherent Distributed Feedback Lasers from Dense Colloidal Quantum Dot Films. Appl. Phys. Lett. 2013, 103, 171104.

(22) Roh, K.; Dang, C.; Lee, J.; Chen, S.; Steckel, J. S.; Coe-Sullivan, S.; Nurmikko, A. Surface-Emitting Red, Green, and Blue Colloidal Quantum Dot Distributed Feedback Lasers. Opt. Express 2014, 22, 18800-18806.

(23) Klimov, V. I.; Ivanov, S. A.; Nanda, J.; Achermann, M.; Bezel, I.; McGuire, J. A.; Piryatinski, A. Single-Exciton Optical Gain in Semiconductor Nanocrystals. Nature 2007, 447, 441-446.

(24) Sewall, S. L.; Cooney, R. R.; Dias, E. A.; Tyagi, P.; Kambhampati, P. State-Resolved Observation in Real Time of the Structural Dynamics of Multiexcitons in Semiconductor Nanocrystals. Phys. Rev. B: Condens. Matter Mater. Phys. 2011, 84, 235304.

(25) Sewall, S. L.; Cooney, R. R.; Anderson, K. E.; Dias, E. A.; Kambhampati, P. State-to-State Exciton Dynamics in Semiconductor Quantum Dots. Phys. Rev. B: Condens. Matter Mater. Phys. 2006, 74, 235328 .

(26) Sewall, S. L.; Cooney, R. R.; Anderson, K. E.; Dias, E. A.; Sagar, D.; Kambhampati, P. State-Resolved Studies of Biexcitons and Surface Trapping Dynamics in Semiconductor Quantum Dots. J. Chem. Phys. 2008, 129, 084701. 
(27) Knappenberger, K. L.; Wong, D. B.; Xu, W.; Schwartzberg, A. M.; Wolcott, A.; Zhang, J. Z.; Leone, S. R. Excitation-Wavelength Dependence of Fluorescence Intermittency in Cdse Nanorods. ACS Nano 2008, 2, 2143-2153.

(28) Knappenberger, K. L.; Wong, D. B.; Romanyuk, Y. E.; Leone, S. R. Excitation Wavelength Dependence of Fluorescence Intermittency in Cdse/Zns Core/Shell Quantum Dots. Nano Lett. 2007, 7, 38693874.

(29) Klimov, V. I. Nanocrystal Quantum Dots, 2nd ed.; CRC Press: Boca Raton, FL, 2010.

(30) Kambhampati, P. Hot Exciton Relaxation Dynamics in Semiconductor Quantum Dots: Radiationless Transitions on the Nanoscale. J. Phys. Chem. C 2011, 115, 22089-22109.

(31) Shevchenko, E. V.; Talapin, D. V.; Kotov, N. A.; O’Brien, S.; Murray, C. B. Structural Diversity in Binary Nanoparticle Superlattices. Nature 2006, 439, 55-59.

(32) Krause, M. M.; Kambhampati, P. Linking Surface Chemistry to Optical Properties of Semiconductor Nanocrystals. Phys. Chem. Chem. Phys. 2015, 17, 18882-18894.

(33) Kambhampati, P. On the Kinetics and Thermodynamics of Excitons at the Surface of Semiconductor Nanocrystals: Are There Surface Excitons? Chem. Phys. 2015, 446, 92-107. 